\title{
Superconductivity of $\mathrm{KFe}_{2} \mathrm{As}_{2}$ Under Pressure: Ab Initio Study of Tetragonal and Collapsed Tetragonal Phases
}

\author{
Andrzej Ptok $^{1}$ (D) $\cdot$ Konrad Jerzy Kapcia ${ }^{1}$ (D) . Małgorzata Sternik ${ }^{1}$ (D) . Przemysław Piekarz ${ }^{1}$ (I)
}

Received: 6 November 2019 / Accepted: 7 February 2020 / Published online: 28 April 2020

(C) The Author(s) 2020

\begin{abstract}
$\mathrm{KFe}_{2} \mathrm{As}_{2}$ is one of the representatives of iron-based superconductors. Many interesting features distinguish this compound from other iron-based superconductors, e.g., a realization of the Pauli limit or an occurrence of the superconducting gap with nodal lines. Moreover, with increasing pressure, the isostructural phase transition from the tetragonal to collapsed tetragonal phase is experimentally observed. We discuss the structural, electronic, and superconducting properties of the $\mathrm{KFe}_{2} \mathrm{As}_{2}$ under pressure using the ab initio density functional theory (DFT) methods. We analyze the untypical properties of this superconductor considering, among others, the Fermi surfaces or the dependence of the anion height from the iron layer on the superconducting critical temperature.
\end{abstract}

Keywords Iron-based superconductors · Isostructural phase transition - DFT calculations · Fermi surface

\section{Introduction}

High-temperature superconductivity in iron-based materials was first observed in 2008 [1]. This discovery opened a period of intensive experimental studies of this class of compounds [2-4]. The unique properties caused by the layered structure [2] and multi-band electronic structure [5, 6] were observed. A typical iron-based superconductor (IBSC) exhibits the multi-gap [6, 7] unconventional superconductivity with the $s_{ \pm}$-wave pairing symmetry [8], i.e., the superconducting gap at different pockets of the Fermi surfaces has opposite signs [9]. In contrast to the other IBSC, the $\mathrm{KFe}_{2} \mathrm{As}_{2}$ compound (K122) possesses

\footnotetext{
Andrzej Ptok

aptok@mmj.pl

Konrad Jerzy Kapcia

konrad.kapcia@ifj.edu.pl

Małgorzata Sternik

malgorzata.sternik@ifj.edu.pl

Przemysław Piekarz

piekarz@wolf.ifj.edu.pl
}

1 Institute of Nuclear Physics, Polish Academy of Sciences, ul. E. Radzikowskiego 152, PL-31342 Kraków, Poland some unusual features. For example, the $d$-wave gap with the nodal line is expected in the superconducting phase [1016]. Moreover, K122 is the Pauli limit superconductor [17], what is in contrast to majority of IBSC [18].

$\mathrm{KFe}_{2} \mathrm{As}_{2}$ is the end-member of the $\mathrm{Ba}_{1-x} \mathrm{~K}_{x} \mathrm{Fe}_{2} \mathrm{As}_{2}$ series. The maximum of critical temperature $T_{c}=38 \mathrm{~K}$ was found at optimal doping $x \approx 0.4$ [19]. However, for a clean $\mathrm{K} 122, T_{c}$ is estimated to be approximately $3.5 \mathrm{~K}[10,20]$. In a contrary to the parent Ba122 compound, K122 does not possess the low-temperature orthorhombic phase and does not show any magnetic order [21].

Usually, the IBSCs exhibit a structural phase transition between the tetragonal and orthorhombic phases by lowering temperature or inducing chemical pressure [22]. $\mathrm{K} 122$ is very special because it crystallizes in the tetragonal structure, even at very low temperatures. What is more interesting, with increasing pressure it exhibits the isostructural phase transition from the tetragonal $(\mathrm{T})$ phase to the collapsed tetragonal (cT) phase [23, 24]. This transition is associated with a strong modification of the lattice parameters without lost of the crystal symmetry. The experimental evidence of the T-cT phase transition has been reported also for other 122 IBSC representatives, e.g., Ca122 [25], Ba122 [26, 27], or Eu122 [28].

Superconducting properties of K122 can be changed by increasing the external pressure. In particular, $T_{c}$ shows nonmonotonic dependence on pressure [23, 24, 29-32], with a minimum near $1.8 \mathrm{GPa}$ [32]. Moreover, $T_{c}$ exhibits an 
universal behavior of the $\mathrm{V}$-shape dependence in the weak pressure regime [33]. It has been reported not only in a case of K122 [32] but also for Rb122 [33] and Cs122 [34].

In the present work, we discuss the effect of the pressure on the superconducting phase of K122. We present it in the context of electronic properties obtained by the ab initio density functional theory (DFT) method. The paper is organized as follows. In Section 2, we show the numerical results and describe the influence of the external hydrostatic pressure on physical properties of the system related to the isostructural phase transition: main structural properties (Section 2.1), electronic properties (Section 2.2), and superconducting properties (Section 2.3). Additionally, in Section 2.4, we discuss our results in the context of other high- $T_{c}$ superconductors. Finally, Section 3 is devoted to conclusions.

\section{Numerical Results and Discussion}

The DFT calculations were performed using the PBE GGA [36] exchange-correlation functional implemented in VASP [37, 38]. They were carried out for conventional cell (cf. Fig. 1a) using the $16 \times 16 \times 8$ Monkhorst-Pack grid of $k$-points [39] and the energy cut-off of $450 \mathrm{eV}$. The crystal structure was optimized using the conjugate gradient technique with the energy convergence criteria set at $10^{-7} \mathrm{eV}$ and $10^{-5} \mathrm{eV}$ for the electronic and ionic iterations, respectively. During calculations, the lattice constants $a$ and $c$ as well as the position of As $\left(z_{A s}\right)$ were optimized. The optimization of the crystal structure was repeated for several hydrostatic pressures and the obtained results are presented in Fig. $1 b$ and $c$.

\subsection{Isostructural Phase Transition}

K122 crystallizes in the tetragonal $14 / \mathrm{mmm}$ structure (space group: 139) (Fig. 1a). Under normal conditions, the structural parameters of the K122 tetragonal phase were specified as $a=b=3.842 \AA, c=13.861 \AA$, and $z_{A s}=$ 0.3525 [42]. Imposed hydrostatic pressure leads to the isostructural phase transition. With increasing pressure, the lattice constant $c$ decreases monotonically with the largest reduction of its value around the critical pressure $p_{c} \approx$ $14 \mathrm{GPa}$ (Fig. 1b). In contrast, the lattice constant $a$ starts to increase at pressure around $10 \mathrm{GPa}$ and it is suddenly enlarged around $p_{c}$. At the same time, the dramatic changes of the angle $\alpha$, defined in Fig. 1a, and the distance of the As atoms from the Fe plane $\left(h_{A s}\right)$ are also observed. Above $p_{c}$, $\alpha$ and $h_{A s}$ reach the lowest and highest value, respectively. The obtained dependencies of the lattice parameters are in agreement with the experimental data [23].

The main source of the isostructural phase transition in 122 compounds is the formation of the As-As bonds between initially non-bonded As atoms caused by the overlap of the $4 p_{z}$ orbitals due to the imposed pressure [4345]. Our findings are in a good agreement with this scenario. An emergence of the As-As bonding with increasing pressure is illustrated in Fig. 2, where the isocharge surfaces around the As atoms for several pressures are presented. The large As-As distance of non-bonded atoms is easily shortened by the pressure up to the value for which the
Fig. 1 (a) Tetragonal (I4/mmm) structure of $\mathrm{KFe}_{2} \mathrm{As}_{2}$. The image was rendered using VESTA software [35]. Pressure dependencies of (b) lattice constants $a$ and $c$ and (c) angle $\alpha$ between two $\mathrm{Fe}-\mathrm{As}$ bonds [indicated in panel (a)] and distance $h_{\mathrm{As}}$ of the As atom from the Fe plane

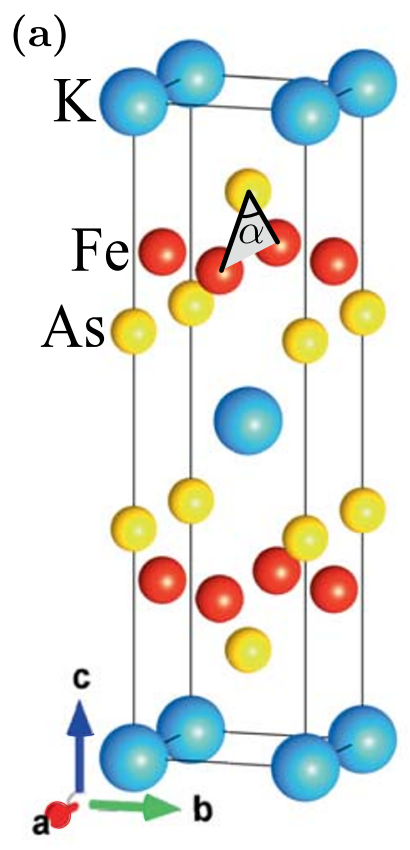

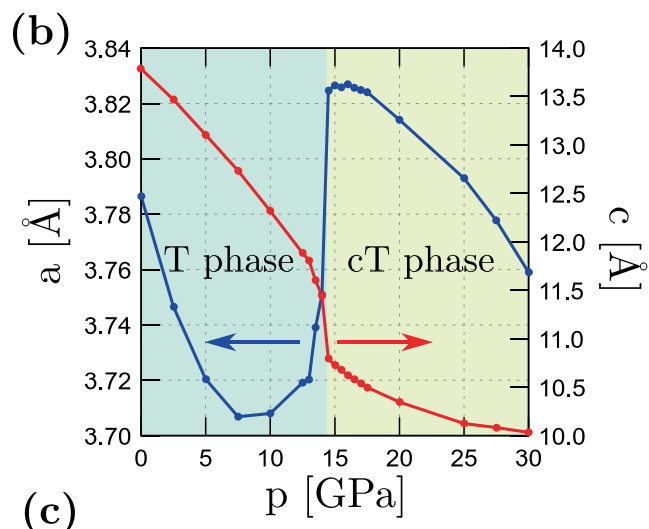

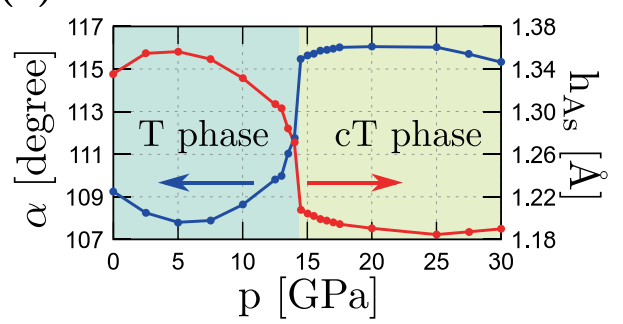


Fig. 2 Formation of the As-As bond with the increasing pressure visualized by the evolution of the isocharge surface (yellow one) corresponding to As atoms (cf. with Fig. 1(a)). The tetragonal K122 conventional cell in the same scale is shown for a comparison. The image was rendered using XCRYSDEN software [40]

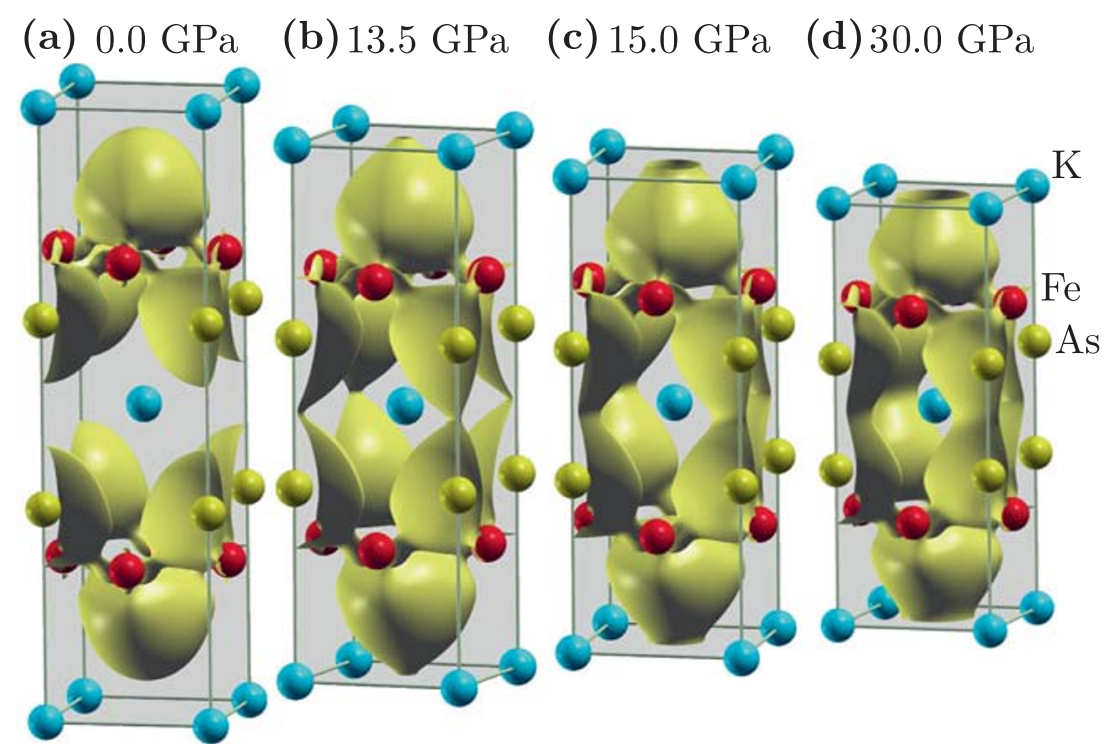

overlap of orbitals is possible. When it happens, the $p_{z}$ orbitals of the As atoms cover partially the $p_{z}$ orbitals of the As atoms from neighboring FeAs layers [46]. It means that the initially separated isosurfaces bring together creating "hourglass" shapes as it is shown in Fig. 2. Consequently, the $\mathrm{Fe}-\mathrm{As}$ bonds become weaker and the lattice parameter $a$ increases, whereas $c$ decreases at the phase transition.

\subsection{Electronic Properties}

The spectra measured by the angle resolved photoemission spectroscopy (ARPES) showed the existence of three hole and four electron pockets centered at the $\Gamma$ and the $\mathrm{X}$ point, respectively [20, 47-49]. To achieve a consistency of these results with our DFT calculations, it is necessary to shift the theoretical value of the Fermi level of approximately $75 \mathrm{meV}$ [46]. Such a small shift corresponds to the change of the average number of particles by $\delta n \approx 0.075$ [50], what does not change qualitatively the obtained electronic structure.

The resulting Fermi surfaces (FS) are shown in Fig. 3. In the absence of pressure, the FS of K122 have a characteristic cylindrical shape (Fig. 3a), also observed for other IBSC [5, 51-53]. Additionally, the FS calculated for $p=0 \mathrm{GPa}$ very well reproduces the experimental results obtained by ARPES [20, 49]. The increasing pressure causes the significant modification of the FS around the Brillouin zone boundary (cf. Fig. 3b, c). The pocket observed close to the $X$ point at $p=0 \mathrm{GPa}$ is shifted to the zone boundary and changes its character from the hole-like to the electron-like at higher pressures [23].

Between 13.5 and $15 \mathrm{GPa}$, the topology of the FS alters indicating that the $\mathrm{T}-\mathrm{cT}$ structural phase transition is accompanied by the Lifshitz transition [54]. Typically, it is generated by doping [14, 20, 55-61] or external magnetic field [62-64]. In the case of K122, the Lifshitz transition
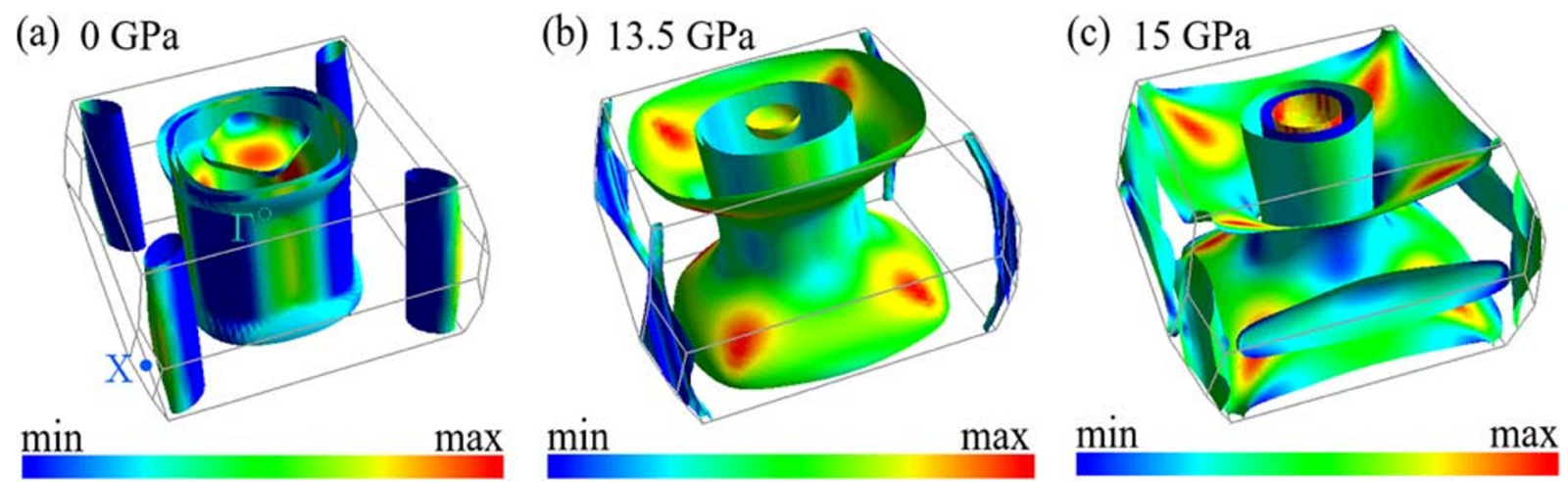

Fig. 3 Fermi surface of the K122 for several pressures (as labeled). Color corresponds to the Fermi velocity. On panel (a), $\Gamma$ - and X-points of the Brillouin zone are indicated. The image was rendered using FERMISURFER software [41] 
is induced by the pressure, which leads to the strong modification of the band structure around $p_{c}$ [46]. The FS shape is also strongly changed as it is shown in Fig. $3 \mathrm{~b}$ and c. The largest modification of the FS is observed at a location of the smallest Fermi velocity $\left(\mathbf{v}_{F} \sim \partial E_{k} / \partial k\right)$.

The Lifshitz transition is correlated with the modification of the FS topology and also with the alteration of orbitals contribution to the FS. A perfect nesting between electron and hole pockets of the FS [65] reported in many IBSC [3, 66-71] is usually observed in the absence of the pressure. In the case of K122, by contrast, the perfect nesting is not observed for pressures lower than $p_{c}$. Thus, one cannot expect a realization of any magnetic order or nematic phases in this compound [72, 73]. This can be changed by the external pressure, high enough to transform system into the cT phase. For $p>p_{c}$, the FS shape $[23,74]$ is very similar to those in the magnetic $\mathrm{YFe}_{2} \mathrm{Ge}_{2}$ [75] or $\mathrm{YRu}_{2} \mathrm{Ge}_{2}$ [76] compounds. Thus, in the cT phase of $\mathrm{K} 122$, one can also expect a realization of a magnetic order. It should be underlined that, in general, the magnetism and superconductivity are competitive phenomena.

\subsection{Superconducting Properties}

At present, it is believed that the antiferromagnetic spin fluctuations $[7,22,77-80]$ can be a source of the $s_{ \pm}$ superconductivity [80-83] in the IBSC. On the other hand, the experiments performed for K122 at ambient pressure $(p=0)$ show that this material is characterized by the nodal $d$-wave-type gap [10-16, 84-86]. However, with increasing pressure, the gap symmetry can be changed from $d$-wave to $s_{ \pm}$-wave [87].

As it was mentioned previously, the lattice parameters change under external pressure (cf. Fig. 1). In particular, the pressure modifies the distance between the As atoms and the Fe layer ( $h_{\mathrm{As}}$ at Fig. 1c). For known IBSC, the apparent correlation between the anion (As, Se, and others) height from the iron layer and the superconducting critical temperature was already reported [88]. Figure 4 shows the critical temperature $T_{c}$ for several IBSC in relation to the distance between anions and $\mathrm{Fe}$ layers using data presented in Ref. [88]. We supplemented this plot by the new data for K122 at zero and $30 \mathrm{GPa}$ pressures (blue open circles in Fig. 4). The maximal value of $T_{c}$ is observed for the anion-the Fe layer distance of approximately $1.4 \AA$. The FeSe layered material is a nice example of the beneficial influence of the external pressure on the critical temperature (red line in Fig. 4). In this case, a pressure increased from 0 to $4 \mathrm{GPa}$ leads to a decrease of $h_{\mathrm{Se}}$ and an increase of $T_{c}$. Similar effect is observed for the $(\mathrm{Ba}, \mathrm{K}) 122$ family (dashed blue line in Fig. 4) but the source of $h_{\mathrm{As}}$ modification is different. The highest $T_{c}$ was found when $\mathrm{K}$ doping leads to $h_{\mathrm{As}} \approx 1.4 \AA$ (pink diamond in Fig. 4).

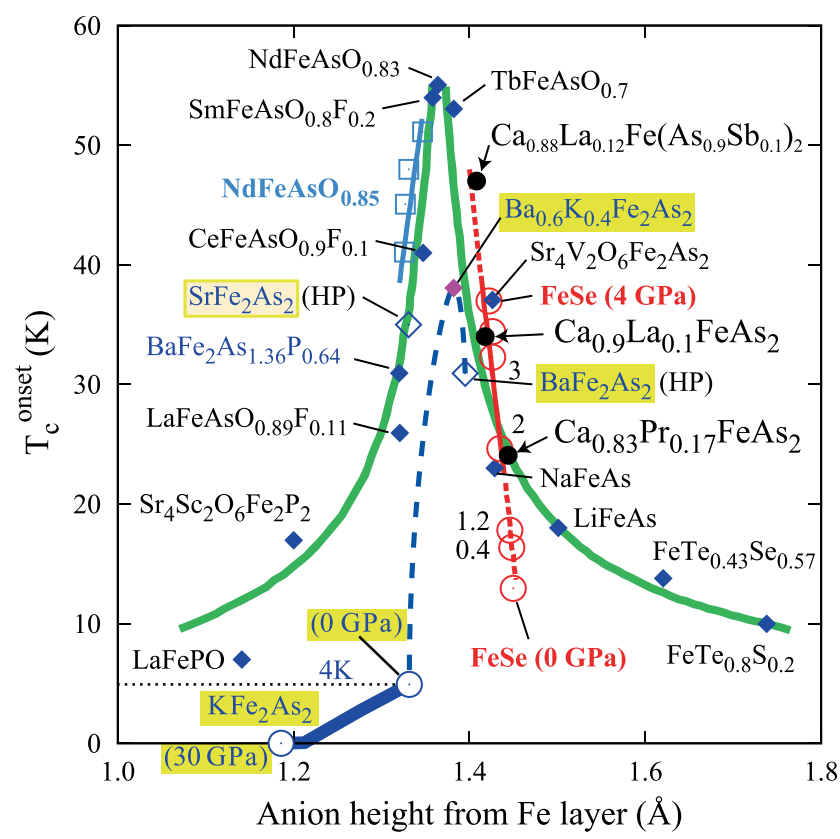

Fig. 4 Relation between the anion height from the iron layer and the critical temperature. Discussed results for $\mathrm{KFe}_{2} \mathrm{As}_{2}$ under pressure are shown by solid blue line. Compounds from $\mathrm{Ba}_{1-x} \mathrm{~K}_{x} \mathrm{Fe}_{2} \mathrm{As}_{2}$ family are marked by yellow frames, while the optimal doping is denoted by pink diamond. Experimental data are adopted from Ref. [88]

To compare the K122 compound with other IBSC, we analyzed the dependence of $h_{\mathrm{As}}$ on pressure (Fig. 1c) and the experimental value of $T_{c}$ [23, 24, 29-32]. Additionally, we assume that the superconductivity vanishes in the highpressure phase what is in accordance with the predicted occurrence of magnetic order in the cT phase. The obtained solid blue line shown in Fig. 4 diverges from the solid green line determined for other materials due to the fact that $\mathrm{K} 122$ has much smaller $T_{c}$ then the other IBSC with similar $h_{\mathrm{As}}$. Nevertheless, the rate of $T_{c}$ change with $h_{\mathrm{As}}$ is similar as in other IBSC.

The above considerations indicate that a question about a source of pairing in the K122 compound is still open and its explanation is necessary to the proper description of $\mathrm{K} 122$ properties.

\subsection{Comparison with Other High- $T_{c}$ Superconductors}

The isostructural phase transition as well as a disappearance of the superconductivity under pressure should be connected with a modification of the Fermi surface topology, i.e., the Lifshitz transition. This connection was also observed in other high- $T_{c}$ superconductors. During the Lifshitz transition, an appearing band has energy of the order of the cut-off energy of interaction and of the energy gap [89, 90]. In this regime, the superconducting phase is characterized by a superconducting dome (superstripes) with the critical temperature of the optimally doped phase due to a quantum 
resonance called the Fano resonance (or the Feshbach resonance) [91-93] (for a review see also, e.g., Ref. [94]).

Theoretical calculations for the multi-band superconductors show that the change of the character of the electrons from 3D to 2D behavior (which can be observed as a modification of a shape of the Fermi surface, e.g., from cilidricallike to spherical-like) leads to a stabilization of the superconductivity [95-97] due to the emergence of the narrow bands [98]. In such a case, the van Hove singularity plays an important role in tuning of the superconductivity [99, 100]. The manifestation of this behavior is, for instance, an appearance of the superconducting phase in the magicangle graphene superlattices [101, 102], width-dependence of $T_{c}$ of nanofilms $[103,104]$, or room- $T_{c}$ (under external pressure) hydride superconductors $[105,106]$.

The influence of the dimensionality of the system onto superconductivity was also studied in the case of $\mathrm{MgB}_{2}$ [107]. Here, it is worth to mention that in some cases, a reduction of the system dimensionality can lead to stabilization of the unconventional superconducting phases [108]. In the studied case of the K122 under pressure, we observed the opposite behavior to the discussed above. Namely, the pressure leads to increase of the dimensionality of the system and to a disappearance of the superconductivity (cf. Section 2.2).

\section{Summary}

In this work, we discuss the untypical properties of $\mathrm{KFe}_{2} \mathrm{As}_{2}$ according to the results obtained from the ab initio DFT calculations. We showed that the increasing pressure leads to the isostructural phase transition at some critical pressure $p_{c}$ between 13.5 and $15 \mathrm{GPa}$. Around $p_{c}$ the new strong bonding between As atoms emerges. As a consequence, the strong modification of the lattice parameters without lost of the symmetry is observed. We accurately reproduced the experimentally observed lattice constants dependence on pressure. We demonstrated also that the modification of structural parameters leads to the changes of the electronic structure that yield the Lifshitz transition. Additionally, the analysis of the Fermi surface of the cT phase leads us to the opinion that occurrence of some sort of magnetic order in the collapsed tetragonal phase of K122 is possible.

We examined also the relation between the anion height from $\mathrm{Fe}$ layers and the superconducting critical temperature in $\mathrm{KFe}_{2} \mathrm{As}_{2}$ compared with the other IBSC. The distance of $1.34 \AA$ at zero-pressure should locate K122 compound among the superconductors with $T_{c}$ higher than $20 \mathrm{~K}$. However, $\mathrm{KFe}_{2} \mathrm{As}_{2}$ has much smaller $T_{c}$. An imposed pressure decreases both the superconducting critical temperature (to $T_{c}=0 \mathrm{~K}$ in $\mathrm{cT}$ phase) and the distance from the As atom to Fe layers. The resulting $T_{c}$ dependence on the anion height from Fe layers agrees with the relation observed for other iron-based superconductors but the temperature range is different. The described untypical properties together with the observation of the gap function with nodal lines (i.e., with $d$-wave symmetry) make $\mathrm{KFe}_{2} \mathrm{As}_{2}$ a very attractive compound for future studies. In particular, the very important question of the nature of the superconductivity in this compound is still open.

Acknowledgments The authors are thankful to Paweł Jochym, Jan Łażewski, Andrzej M. Oleś, and Krzysztof Parlinski for the very fruitful discussions and comments.

Funding Information This work was financially supported by the National Science Centre (NCN, Poland) under grants UMO-2017/25/B/ST3/02586 (A.P., M.S., and P.P.), and UMO2017/24/C/ST3/00276 (K.J.K.). A.P. and K. J. K. appreciate also founding in the frame of scholarships of the Minister of Science and Higher Education (Poland) for outstanding young scientists (2019 edition, nos. 818/STYP/14/2019 and 821/STYP/14/2019, respectively).

Open Access This article is licensed under a Creative Commons Attribution 4.0 International License, which permits use, sharing, adaptation, distribution and reproduction in any medium or format, as long as you give appropriate credit to the original author(s) and the source, provide a link to the Creative Commons licence, and indicate if changes were made. The images or other third party material in this article are included in the article's Creative Commons licence, unless indicated otherwise in a credit line to the material. If material is not included in the article's Creative Commons licence and your intended use is not permitted by statutory regulation or exceeds the permitted use, you will need to obtain permission directly from the copyright holder. To view a copy of this licence, visit http:// creativecommonshorg/licenses/by/4.0/.

\section{References}

1. Kamihara, Y., Watanabe, T., Hirano, M., Hosono, H.: J. Am. Chem. Soc. 130, 3296 (2008). https://doi.org/10.1021/ ja800073m

2. Stewart, G.R.: Rev. Mod. Phys. 83, 1589 (2011). https://doi.org/10.1103/RevModPhys.83.1589

3. Dai, P.: Rev. Mod. Phys. 87, 855 (2015). https://doi.org/10.1103/ RevModPhys.87.855

4. Hosono, H., Yamamoto, A., Hiramatsu, H., Ma, Y.: Materials today. https://doi.org/10.1016/j.mattod.2017.09.006 (2017)

5. Kordyuk, A.A.: Low Temp. Phys. 38, 888 (2012). https://doi.org/ 10.1063/1.4752092

6. Richard, P., Qian, T., Ding, H.: J. Phys. Condens. Matter 27, 293203 (2015). https://doi.org/10.1088/0953-8984/27/29/ 293203

7. Hirschfeld, P.J., Korshunov, M.M., Mazin, I.I.: Rep. Prog. Phys. 74, 124508 (2011). https://doi.org/10.1088/0034-4885/74/12/ 124508

8. Korshunov, M.M., Eremin, I.: Phys. Rev. B 78, 140509 (2008). https://doi.org/10.1103/PhysRevB.78.140509

9. Ptok, A., Crivelli, D., Kapcia, K.J.: Supercond. Sci. Technol. 28, 045010 (2015). https://doi.org/10.1088/0953-2048/28/4/045010

10. Fukazawa, H., Yamada, Y., Kondo, K., Saito, T., Kohori, Y., Kuga, K., Matsumoto, Y., Nakatsuji, S., Kito, H., Shirage, P.M., Kihou, K., Takeshita, N., Lee, C.H., Iyo, A., Eisaki, H.: J. Phys. 
Soc. Jpn. 78, 083712 (2009). https://doi.org/10.1143/JPSJ.78. 083712

11. Hashimoto, K., Serafin, A., Tonegawa, S., Katsumata, R., Okazaki, R., Saito, T., Fukazawa, H., Kohori, Y., Kihou, K., Lee, C.H., Iyo, A., Eisaki, H., Ikeda, H., Matsuda, Y., Carrington, A., Shibauchi, T.: Phys. Rev. B 82, 014526 (2010). https://doi.org/10.1103/PhysRevB.82.014526

12. Dong, J.K., Zhou, S.Y., Guan, T.Y., Zhang, H., Dai, Y.F., Qiu, X., Wang, X.F., He, Y., Chen, X.H., Li, S.Y.: Phys. Rev. Lett. 104, 087005 (2010). https://doi.org/10.1103/PhysRevLett.104. 087005

13. Okazaki, K., Ota, Y., Kotani, Y., Malaeb, W., Ishida, Y., Shimojima, T., Kiss, T., Watanabe, S., Chen, C.T., Kihou, K., Lee, C.H., Iyo, A., Eisaki, H., Saito, T., Fukazawa, H., Kohori, Y., Hashimoto, K., Shibauchi, T., Matsuda, Y., Ikeda, H., Miyahara, H., Arita, R., Chainani, A., Shin, S.: Science 337, 1314 (2012). https://doi.org/10.1126/science.1222793

14. Xu, N., Richard, P., Shi, X., van Roekeghem, A., Qian, T., Razzoli, E., Rienks, E., Chen, G.F., Ieki, E., Nakayama, K., Sato, T., Takahashi, T., Shi, M., Ding, H.: Phys. Rev. B 88, 220508 (2013). https://doi.org/10.1103/PhysRevB.88.220508

15. Ota, Y., Okazaki, K., Kotani, Y., Shimojima, T., Malaeb, W., Watanabe, S., Chen, C.T., Kihou, K., Lee, C.H., Iyo, A., Eisaki, H., Saito, T., Fukazawa, H., Kohori, Y., Shin, S.: Phys. Rev. B 89, 081103 (2014). https://doi.org/10.1103/PhysRevB.89.081103

16. Kittaka, S., Aoki, Y., Kase, N., Sakakibara, T., Saito, T., Fukazawa, H., Kohori, Y., Kihou, K., Lee, C.H., Iyo, A., Eisaki, H., Deguchi, K., Sato, N.K., Tsutsumi, Y., Machida, K.: J. Phys. Soc. Jpn. 83, 013704 (2014). https://doi.org/10.7566/JPSJ.83. 013704

17. Zocco, D.A., Grube, K., Eilers, F., Wolf, T., Löhneysen, H.v.: Phys. Rev. Lett. 111, 057007 (2013). https://doi.org/10.1103/ PhysRevLett.111.057007

18. Gurevich, A.: Rep. Prog. Phys. 74, 124501 (2011). https://doi.org/10.1088/0034-4885/74/12/124501

19. Rotter, M., Tegel, M., Johrendt, D.: Phys. Rev. Lett. 101, 107006 (2008). https://doi.org/10.1103/PhysRevLett.101.107006

20. Sato, T., Nakayama, K., Sekiba, Y., Richard, P., Xu, Y.M., Souma, S., Takahashi, T., Chen, G.F., Luo, J.L., Wang, N.L., Ding, H.: Phys. Rev. Lett. 103, 047002 (2009). https://doi.org/ 10.1103/PhysRevLett.103.047002

21. Avci, S., Chmaissem, O., Chung, D.Y., Rosenkranz, S., Goremychkin, E.A., Castellan, J.P., Todorov, I.S., Schlueter, J.A., Claus, H., Daoud-Aladine, A., Khalyavin, D.D., Kanatzidis, M.G., Osborn, R.: Phys. Rev. B 85, 184507 (2012). https://doi.org/10.1103/PhysRevB.85.184507

22. Wang, F., Lee, D.H.: Science 332, 200 (2011). https://doi.org/10.1126/science.1200182

23. Nakajima, Y., Wang, R., Metz, T., Wang, X., Wang, L., Cynn, H., Weir, S.T., Jeffries, J.R., Paglione, J.: Phys. Rev. B 91, 060508 (2015). https://doi.org/10.1103/PhysRevB.91.060508

24. Ying, J.J., Tang, L.Y., Struzhkin, V.V., Mao, H.K., Gavriliuk, A.G., Wang, A.F., Chen, X.H., Chen, X.J.: Tripling the critical temperature of $\mathrm{KFe}_{2} \mathrm{As}_{2}$ by carrier switch. arXiv:1501.00330 (2015)

25. Kreyssig, A., Green, M.A., Lee, Y., Samolyuk, G.D., Zajdel, P., Lynn, J.W., Bud'ko, S.L., Torikachvili, M.S., Ni, N., Nandi, S., Leão, J.B., Poulton, S.J., Argyriou, D.N., Harmon, B.N., McQueeney, R.J., Canfield, P.C., Goldman, A.I.: Phys. Rev. B 78, 184517 (2008). https://doi.org/10.1103/PhysRevB.78. 184517

26. Uhoya, W., Stemshorn, A., Tsoi, G., Vohra, Y.K., Sefat, A.S., Sales, B.C., Hope, K.M., Weir, S.T.: Phys. Rev. B 82, 144118 (2010). https://doi.org/10.1103/PhysRevB.82.144118
27. Mittal, R., Mishra, S.K., Chaplot, S.L., Ovsyannikov, S.V., Greenberg, E., Trots, D.M., Dubrovinsky, L., Su, Y., Brueckel, T., Matsuishi, S., Hosono, H., Garbarino, G.: Phys. Rev. B 83, 054503 (2011). https://doi.org/10.1103/PhysRevB.83.054503

28. Uhoya, W., Tsoi, G., Vohra, Y.K., McGuire, M.A., Sefat, A.S., Sales, B.C., Mandrus, D., Weir, S.T.: J. Phys Condens. Matter 22, 292202 (2010). https://doi.org/10.1088/0953-8984/22/29/ 292202

29. Taufour, V., Foroozani, N., Tanatar, M.A., Lim, J., Kaluarachchi, U., Kim, S.K., Liu, Y., Lograsso, T.A., Kogan, V.G., Prozorov, R., Bud'ko, S.L., Schilling, J.S., Canfield, P.C.: Phys. Rev. B 89, 220509 (2014). https://doi.org/10.1103/PhysRevB.89.220509

30. Wang, P.S., Zhou, P., Dai, J., Zhang, J., Ding, X.X., Lin, H., Wen, H.H., Normand, B., Yu, R., Yu, W.: Phys. Rev. B 93, 085129 (2016). https://doi.org/10.1103/PhysRevB.93.085129

31. Wang, B., Matsubayashi, K., Cheng, J., Terashima, T., Kihou, K., Ishida, S., Lee, C.H., Iyo, A., Eisaki, H., Uwatoko, Y.: Phys. Rev. B 94, 020502 (2016). https://doi.org/10.1103/PhysRevB.94. 020502

32. Tafti, F.F., Juneau-Fecteau, A., Delage, M.E., Rene de Cotret, S., Reid, J.P., Wang, A.F., Luo, X.G., Chen, X.H., DoironLeyraud, N., Taillefer, L.: Nat. Phys. 9, 349 (2013). https://doi.org/10.1038/nphys2617

33. Tafti, F.F., Ouellet, A., Juneau-Fecteau, A., Faucher, S., Lapointe-Major, M., Doiron-Leyraud, N., Wang, A.F., Luo, X.G., Chen, X.H., Taillefer, L.: Phys. Rev. B 91, 054511 (2015). https://doi.org/10.1103/PhysRevB.91.054511

34. Tafti, F.F., Clancy, J.P., Lapointe-Major, M., Collignon, C., Faucher, S., Sears, J.A., Juneau-Fecteau, A., Doiron-Leyraud, N., Wang, A.F., Luo, X.G., Chen, X.H., Desgreniers, S., Kim, Y.J., Taillefer, L.: Phys. Rev. B 89, 134502 (2014). https://doi.org/10.1103/PhysRevB.89.134502

35. Momma, K., Izumi, F.: J. Appl. Crystallogr. 44, 1272 (2011). https://doi.org/10.1107/S0021889811038970

36. Perdew, J.P., Burke, K., Ernzerhof, M.: Phys. Rev. Lett. 77, 3865 (1996). https://doi.org/10.1103/PhysRevLett.77.3865

37. Kresse, G., Furthmüller, J.: Comput. Mater. Sci. 6, 15 (1996). https://doi.org/10.1016/0927-0256(96)00008-0

38. Kresse, G., Furthmüller, J.: Phys. Rev. B 54, 11169 (1996). https://doi.org/10.1103/PhysRevB.54.11169

39. Monkhorst, H.J., Pack, J.D.: Phys. Rev. B 13, 5188 (1976). https://doi.org/10.1103/PhysRevB.13.5188

40. Kokalj, A.: Comput. Mater. Sci. 28, 155 (2003). https://doi.org/10.1016/S0927-0256(03)00104-6

41. Kawamura, M.: Comput. Phys. Commun. 239, 197 (2019). https://doi.org/10.1016/j.cpc.2019.01.017

42. Sándor, R., Hans-Uwe, S., Naturforsch, Z.: B Chem. Sci. 36, 1668 (1981). 10.1515/znb-1981-1234

43. Yildirim, T.: Phys. Rev. Lett. 102, 037003 (2009). https://doi.org/10.1103/PhysRevLett.102.037003

44. Kasinathan, D., Schmitt, M., Koepernik, K., Ormeci, A., Meier, K., Schwarz, U., Hanfland, M., Geibel, C., Grin, Y., LeitheJasper, A., Rosner, H.: Phys. Rev. B 84, 054509 (2011). https://doi.org/10.1103/PhysRevB.84.054509

45. Stavrou, E., Chen, X.J., Oganov, A.R., Wang, A.F., Yan, Y.J., Luo, X.G., Chen, X.H., Goncharov, A.F.: Sci. Rep. 5, 9868 (2015). https://doi.org/10.1038/srep09868

46. Ptok, A., Sternik, M., Kapcia, K.J., Piekarz, P.: Phys. Rev. B 99, 134103 (2019). https://doi.org/10.1103/PhysRevB.99. 134103

47. Yoshida, T., Nishi, I., Fujimori, A., Yi, M., Moore, R., Lu, D.H., Shen, Z.X., Kihou, K., Shirage, P., Kito, H., Lee, C., Iyo, A., Eisaki, H., Harima, H.: J. Phys. Chem. Solids 72, 465 (2011). https://doi.org/10.1016/j.jpcs.2010.10.064 
48. Terashima, T., Kurita, N., Kimata, M., Tomita, M., Tsuchiya, S., Imai, M., Sato, A., Kihou, K., Lee, C.H., Kito, H., Eisaki, H., Iyo, A., Saito, T., Fukazawa, H., Kohori, Y., Harima, H., Uji, S.: Phys. Rev. B 87, 224512 (2013). https://doi.org/10.1103/PhysRevB.87. 224512

49. Yoshida, T., Ideta, S.i., Nishi, I., Fujimori, A., Yi, M., Moore, R., Mo, S.K., Lu, D., Shen, Z.X., Hussain, Z., Kihou, K., Lee, C.H., Iyo, A., Eisaki, H., Harima, H.: Front. Phys. 2, 17 (2014). https://doi.org/10.3389/fphy.2014.00017

50. Wang, Y., Kreisel, A., Zabolotnyy, V.B., Borisenko, S.V., Büchner, B., Maier, T.A., Hirschfeld, P.J., Scalapino, D.J.: Phys. Rev. B 88, 174516 (2013). https://doi.org/10.1103/PhysRevB.88. 174516

51. Borisenko, S.V., Zabolotnyy, V.B., Evtushinsky, D.V., Kim, T.K., Morozov, I.V., Yaresko, A.N., Kordyuk, A.A., Behr, G., Vasiliev, A., Follath, R., Büchner, B.: Phys. Rev. Lett. 105, 067002 (2010). https://doi.org/10.1103/PhysRevLett.105.067002

52. Kordyuk, A.A., Zabolotnyy, V.B., Evtushinsky, D.V., Yaresko, A.N., Büchner, B., Borisenko, S.V.: J. Supercond. Nov. Magn. 26, 2837 (2013). https://doi.org/10.1007/s10948-013-2210-8

53. Derondeau, G., Bisti, F., Kobayashi, M., Braun, J., Ebert, H., Rogalev, V.A., Shi, M., Schmitt, T., Ma, J., Ding, H., Strocov, V.N.: J. Minár, Sci. Rep. 7, 8787 (2017). https://doi.org/10.1038/s41598-017-09480-y

54. Lifshitz, I.M.: Zh. Eksp. Teor. Fiz. 38, 1569 (1960). [Sov. Phys. JETP 11, 1130-1135 (1960)]

55. Liu, C., Kondo, T., Fernandes, R.M., Palczewski, A.D., Mun, E.D., Ni, N., Thaler, A.N., Bostwick, A., Rotenberg, E., Schmalian, J., Bud'ko, S.L., Canfield, P.C., Kaminski, A.: Nat. Phys. 6, 419 (2010). https://doi.org/10.1038/nphys1656

56. Nakayama, K., Sato, T., Richard, P., Xu, Y.M., Kawahara, T., Umezawa, K., Qian, T., Neupane, M., Chen, G.F., Ding, H., Takahashi, T.: Phys. Rev. B 83, 020501 (2011). https://doi.org/10.1103/PhysRevB.83.020501

57. Malaeb, W., Shimojima, T., Ishida, Y., Okazaki, K., Ota, Y., Ohgushi, K., Kihou, K., Saito, T., Lee, C.H., Ishida, S., Nakajima, M., Uchida, S., Fukazawa, H., Kohori, Y., Iyo, A., Eisaki, H., Chen, C.T., Watanabe, S., Ikeda, H., Shin, S.: Phys. Rev. B 86, 165117 (2012). https://doi.org/10.1103/PhysRevB. 86.165117

58. Liu, Y., Lograsso, T.A.: Phys. Rev. B 90, 224508 (2014). https://doi.org/10.1103/PhysRevB.90.224508

59. Khan, S.N., Johnson, D.D.: Phys. Rev. Lett. 112, 156401 (2014). https://doi.org/10.1103/PhysRevLett.112.156401

60. Hodovanets, H., Liu, Y., Jesche, A., Ran, S., Mun, E.D., Lograsso, T.A., Bud'ko, S.L., Canfield, P.C.: Phys. Rev. B 89, 224517 (2014). https://doi.org/10.1103/PhysRevB.89.224517

61. Cho, K., Kończykowski, M., Teknowijoyo, S., Tanatar, M.A., Liu, Y., Lograsso, T.A., Straszheim, W.E., Mishra, V., Maiti, S., Hirschfeld, P.J., Prozorov, R.: Sci. Adv. 2. https://doi.org/10.1126/sciadv.1600807 (2016)

62. Pfau, H., Daou, R., Lausberg, S., Naren, H.R., Brando, M., Friedemann, S., Wirth, S., Westerkamp, T., Stockert, U., Gegenwart, P., Krellner, C., Geibel, C., Zwicknagl, G., Steglich, F.: Phys. Rev. Lett. 110, 256403 (2013). https://doi.org/10.1103/PhysRevLett.110.256403

63. Ptok, A., Kapcia, K.J., Cichy, A., Oleś, A.M., Piekarz, P.: Sci. Rep. 7, 41979 (2017). https://doi.org/10.1038/srep41979

64. Ptok, A.: Acta Phys. Pol. A 135, 55 (2018). https://doi.org/10.12693/APhysPolA.135.55

65. Chubukov, A.V., Efremov, D.V., Eremin, I.: Phys. Rev. B 78, 134512 (2008). https://doi.org/10.1103/PhysRevB.78.134512

66. Winiarski, M.J., Samsel-Czekała, M., Ciechan, A.: EPL 100, 47005 (2012). https://doi.org/10.1209/0295-5075/100/47005
67. Bao, W., Qiu, Y., Huang, Q., Green, M.A., Zajdel, P., Fitzsimmons, M.R., Zhernenkov, M., Chang, S., Fang, M., Qian, B., Vehstedt, E.K., Yang, J., Pham, H.M., Spinu, L., Mao, Z.Q.: Phys. Rev. Lett. 102, 247001 (2009). https://doi.org/10.1103/ PhysRevLett.102.247001

68. Winiarski, M., Samsel-Czekała, M., Ciechan, A.: J. Alloy. Comp. 566, 187 (2013). https://doi.org/10.1016/j.jallcom.2013. 02.106

69. Rodriguez, E.E., Stock, C., Zajdel, P., Krycka, K.L., Majkrzak, C.F., Zavalij, P., Green, M.A.: Phys. Rev. B 84, 064403 (2011). https://doi.org/10.1103/PhysRevB.84.064403

70. Ciechan, A., Winiarski, M.J., Samsel-Czekała, M.: Intermetallics 41, 44 (2013). https://doi.org/10.1016/j.intermet.2013. 04.015

71. Mou, D., Kong, T., Meier, W.R., Lochner, F., Wang, L.L., Lin, Q., Wu, Y., Bud'ko, S.L., Eremin, I., Johnson, D.D., Canfield, P.C., Kaminski, A.: Phys. Rev. Lett. 117, 277001 (2016). https://doi.org/10.1103/PhysRevLett.117.277001

72. Chubukov, A.V., Khodas, M., Fernandes, R.M.: Phys. Rev. X 6, 041045 (2016). https://doi.org/10.1103/PhysRevX.6.041045

73. Li, T., Su, Y.: J. Phys. Condens. Matter 29, 425603 (2017). https://doi.org/10.1088/1361-648X/aa85f4

74. Tresca, C., Profeta, G.: Phys. Rev. B 95, 165129 (2017). https://doi.org/10.1103/PhysRevB.95.165129

75. Chen, J., Semeniuk, K., Feng, Z., Reiss, P., Brown, P., Zou, Y., Logg, P.W., Lampronti, G.I., Grosche, F.M.: Phys. Rev. Lett. 116, 127001 (2016). https://doi.org/10.1103/PhysRevLett.116. 127001

76. Chajewski, G., Samsel-Czekała, M., Hackemer, A., Wiśniewski, P., Pikul, A., Kaczorowski, D.: Physica B 536, 767 (2018). https://doi.org/10.1016/j.physb.2017.10.063

77. Suzuki, K., Usui, H., Kuroki, K.: Phys. Rev. B 84, 144514 (2011). https://doi.org/10.1103/PhysRevB.84.144514

78. Li, W., Li, J., Zhu, J.X., Chen, Y., Ting, C.S.: EPL 99, 57006 (2012). https://doi.org/10.1209/0295-5075/99/57006

79. Bang, Y., Stewart, G.R.: J. Phys. Condens. Matter 29, 123003 (2017). https://doi.org/10.1088/1361-648X/aa564b

80. Mazin, I.I., Singh, D.J., Johannes, M.D., Du, M.H.: Phys. Rev. Lett. 101, 057003 (2008). https://doi.org/10.1103/PhysRevLett. 101.057003

81. Kuroki, K., Onari, S., Arita, R., Usui, H., Tanaka, Y., Kontani, H., Aoki, H.: Phys. Rev. Lett. 101, 087004 (2008). https://doi.org/10.1103/PhysRevLett.101.087004

82. Ikeda, H., Arita, R., Kuneš, J.: Phys. Rev. B 81, 054502 (2010). https://doi.org/10.1103/PhysRevB.81.054502

83. Wang, Y., Berlijn, T., Hirschfeld, P.J., Scalapino, D.J., Maier, T.A.: Phys. Rev. Lett. 114, 107002 (2015). https://doi.org/10.1103/PhysRevLett.114.107002

84. Reid, J.P., Tanatar, M.A., Juneau-Fecteau, A., Gordon, R.T., de Cotret, S.R., Doiron-Leyraud, N., Saito, T., Fukazawa, H., Kohori, Y., Kihou, K., Lee, C.H., Iyo, A., Eisaki, H., Prozorov, R., Taillefer, L.: Phys. Rev. Lett. 109, 087001 (2012). https://doi.org/10.1103/PhysRevLett.109.087001

85. Thomale, R., Platt, C., Hanke, W., Hu, J., Bernevig, B.A.: Phys. Rev. Lett. 107, 117001 (2011). https://doi.org/10.1103/ PhysRevLett.107.117001

86. Watanabe, D., Yamashita, T., Kawamoto, Y., Kurata, S., Mizukami, Y., Ohta, T., Kasahara, S., Yamashita, M., Saito, T., Fukazawa, H., Kohori, Y., Ishida, S., Kihou, K., Lee, C.H., Iyo, A., Eisaki, H., Vorontsov, A.B., Shibauchi, T., Matsuda, Y.: Phys. Rev. B 89, 115112 (2014). https://doi.org/10.1103/ PhysRevB.89.115112

87. Guterding, D., Backes, S., Jeschke, H.O., Valentí, R.: Phys. Rev. B 91, 140503 (2015). https://doi.org/10.1103/PhysRevB.91.140503 
88. Kang, C.J., Birol, T., Kotliar, G.: Phys. Rev. B 95, 014511 (2017). https://doi.org/10.1103/PhysRevB.95.014511

89. Perali, A., Bianconi, A., Lanzara, A., Saini, N.L.: Solid State Commun. 100, 181 (1996). https://doi.org/10.1016/00381098(96)00373-0

90. Valletta, A., Bianconi, A., Perali, A., Saini, N.L.: Z. Phys. B 104, 707 (1997). https://doi.org/10.1007/s002570050513

91. Caivano, R., Fratini, M., Poccia, N., Ricci, A., Puri, A., Ren, Z.A., Dong, X.L., Yang, J., Lu, W., Zhao, Z.X., Barba, L., Bianconi, A.: Supercond. Sci. Technol. 22, 014004 (2008). https://doi.org/10.1088/0953-2048/22/1/014004

92. Innocenti, D., Caprara, S., Poccia, N., Ricci, A., Valletta, A., Bianconi, A.: Supercond. Sci. Technol. 24, 015012 (2010). https://doi.org/10.1088/0953-2048/24/1/015012

93. Mazziotti, M.V., Valletta, A., Campi, G., Innocenti, D., Perali, A., Bianconi, A.: EPL 118, 37003 (2017). https://doi.org/10.1209/0295-5075/118/37003

94. Bianconi, A.: Nat. Phys. 9, 536 (2013). https://doi.org/10.1038/ nphys 2738

95. Bianconi, A.: J. Supercond. 18, 625 (2005). https://doi.org/10. 1007/s10948-005-0047-5

96. Angilella, G.G.N., Bianconi, A., Pucci, R.: J. Supercond. 18, 619 (2005). https://doi.org/10.1007/s10948-005-0049-3

97. Innocenti, D., Poccia, N., Ricci, A., Valletta, A., Caprara, S., Perali, A., Bianconi, A.: Phys. Rev. B 82, 184528 (2010). https://doi.org/10.1103/PhysRevB.82.184528

98. Micnas, R., Ranninger, J., Robaszkiewicz, S.: Rev. Mod. Phys. 62, 113 (1990). https://doi.org/10.1103/RevModPhys.62.113

99. Cichy, A., Ptok, A.: Phys. Rev. A 97, 053619 (2018). https://doi.org/10.1103/PhysRevA.97.053619
100. Ptok, A., Kapcia, K.J., Rodríguez, K.: Phys. Rev. Materials 2, 024801 (2018). https://doi.org/10.1103/PhysRevMaterials. 2.024801

101. Cao, Y., Fatemi, V., Fang, S., Watanabe, K., Taniguchi, T., Kaxiras, E., Jarillo-Herrero, P.: Nature 556, 43 (2018). https://doi.org/10.1038/nature26160

102. Cao, Y., Fatemi, V., Demir, A., Fang, S., Tomarken, S.L., Luo, J.Y., Sanchez-Yamagishi, J.D., Watanabe, K., Taniguchi, T., Kaxiras, E., Ashoori, R.C., Jarillo-Herrero, P.: Nature 556, 80 (2018). https://doi.org/10.1038/nature26154

103. Guo, Y., Zhang, Y.F., Bao, X.Y., Han, T.Z., Tang, Z., Zhang, L.X., Zhu, W.G., Wang, E.G., Niu, Q., Qiu, Z.Q., Jia, J.F., Zhao, Z.X., Xue, Q.K.: Science 306, 1915 (2004). https://doi.org/10.1126/science.1105130

104. Wójcik, P., Zegrodnik, M.: J. Phys. Condens. Matter 26, 455302 (2014). https://doi.org/10.1088/0953-8984/26/45/455302

105. Bianconi, A., Jarlborg, T.: EPL 112, 37001 (2015). https://doi.org/10.1209/0295-5075/112/37001

106. Jarlborg, T., Bianconi, A.: Sci. Rep. 6, 24816 (2016). https://doi.org/10.1038/srep24816

107. Bianconi, A., Castro, D.D., Agrestini, S., Campi, G., Saini, N.L., Saccone, A., Negri, S.D., Giovannini, M.: J. Phys. Condens. Matter 13, 7383 (2001). https://doi.org/10.1088/0953-8984/13/ $33 / 318$

108. Ptok, A.: J. Phys,. Condens. Matter 29, 475901 (2017). https://doi.org/10.1088/1361-648x/aa928d

Publisher's Note Springer Nature remains neutral with regard to jurisdictional claims in published maps and institutional affiliations. 